\title{
LLX. On the relation of the sensitiveness of the ear to pitch, investigated by a new method
}

\section{Lord Rayleigh O.M. P.R.S.}

To cite this article: Lord Rayleigh O.M. P.R.S. (1907) LLX. On the relation of the sensitiveness of the ear to pitch, investigated by a new method , Philosophical Magazine Series 6, 14:83, 596-604, DOI: $10.1080 / 14786440709463721$

To link to this article: http://dx.doi.org/10.1080/14786440709463721

Published online: 16 Apr 2009.

Submit your article to this journal $₫$

Џ Article views: 5

Q View related articles $\square$

Citing articles: 2 View citing articles 5 
The surface-tension calculated from these measurements was (temp. $=30^{\circ} \mathrm{C}$.) found to be $77 \cdot 0$ dynes per $\mathrm{cm}$.

Another plate, not so sharp, gave $76 \cdot 3$ dynes per $\mathrm{cm}$.

A third, in taking which the wide tube happened to be out of the vertical, gave no definite result on account of the uncertainty of the value of the curvature of the large meniscus. The value of the surface-tension was, however, estimated to lie between 75 and 77 dynes per $\mathrm{cm}$.

The accepted value for the surface-tension of clean distilled water at $30^{\circ} \mathrm{C} .=71 \cdot 3$ dynes per cm. (T. P. Hall, Phil. Mag. 1893). The method described in this article gives results which are fairly self-consistent. The cause of the difference has as yet not been found and is under investigation.

The experiments and observations recorded in this note were made at the Physical Laboratory of the Presidency College, Madras.

IIX. On the Relation of the Sensitiveness of the Ear to Pitch, investigated by a new Method. By Lord RAYLEIGH, O.M., P.R.S.*

T $N$ a former research $+\mathrm{I}$ examined the sensitiveness of the ear to sounds of different pitch with results which were thos summarized :-

$$
\begin{array}{rrrr}
e^{\prime}, & \text { frequency } & =256, & s=6.0 \times 10^{-9}, \\
a^{\prime}, & \quad & =384, & s=4.6 \times 10^{-4}, \\
c^{\prime \prime}, & , \quad & =512, & s=4.6 \times 10^{-9},
\end{array}
$$

no reliable distinction appearing between the two last numbers. "Even the distinction between $6 \cdot 0$ and $4 \cdot 6$ should be accepted with reserve; so that the comparison must not be taken to prove much more than that the condensation necessary for audibility varies but slowly in the singly dashed octave." Here $s$ denotes the condensation (or rarefaction) which in one respect is a maximum and in another a minimum. It is the maximum condensation which occurs during the course of the vibration, but the vibration (and $s$ with it) is the minimum capable of impressing the ear in a progressive wave. The method employed depended upon a knowledge of the rate at which energy was emitted from a resonator under excitation by a freely vibrating tuning-

* Communicated by the Author.

† Phil. Mag. xxxviii. p. 365, 1894: Scientific Papers, iv. p. 125. 
fork. The amplitude of vibration of the prongs of the fork was under continuous observation with the aid of a microscope. From this could be inferred the energy in the fork at any time and the rate at which it was lost. The loss was greatest when the resonator was in action, and the excess was taken to represent the energy converted into sound. From this again the condensation in the progressive waves at a given distance could be calculated. It was remarked that the numbers thus obtained were "somewhat of the nature of upper limits, for they depend upon the assumption that all the dissipation due to the resonator represents production of sound. This may not be strictly the case even with the moderate amplitudes here in question, but the uncertainty is far less than in the case of resonators or organ-pipes caused to speak by wind."

In a careful re-examination of this question, M. Wien *, working with the telephone, finds not only a still higher degree of sensitiveness but a much more rapid variation with pitch. In the following extract from his table xiv., $\mathrm{N}$ represents the frequency and $\Delta$ the proportional excess of pressure, equal to $\gamma s$, where $\gamma$ is the ratio of specific heats of air $(1 \cdot 41)$. The higher degree of sensitiveness may be

\begin{tabular}{|c|c|c|c|}
\hline N. & $\Delta$. & N. & $\Delta$. \\
\hline 50 & $1.6 \times 10^{-\tau}$ & 1600 & $1.4 \times 10^{-11}$ \\
100 & $1.1 \times 10^{-8}$ & 3200 & $1.4 \times 10^{-11}$ \\
200 & $1.0 \times 10^{-9}$ & 6100 & $2.3 \times 10^{-11}$ \\
400 & $1.2 \times 10^{-10}$ & 12800 & $8.0 \times 10^{-11}$ \\
800 & $2.3 \times 10^{-11}$ & & \\
\hline
\end{tabular}

partly explained by the greater precautions taken to ensure silence and by the sounds under observation being rendered intermittent ; or, on the other hand, my estimates of sensitiveness may have been too low in consequence of the already named assumption that all the excess of damping due to the resonator represented produetion of sound. With respect to the dependence on pitch, Wien remarks that my own observations $\dagger$ on the minimum current in the telephone

* Pflüger's Arch. xcvii. p. 1, 1903.

† Phil: Mag. xxxviii. p. 285, 1894; Scientific Papers, iv. p. 109. 
necessary for audibility at various frequencies, support his view of the question. Certainly this is their tendency. At the time when these observations were made the whole modus operandi of the telephone was still involved in doubt, and my object in these observations was rather to elucidate the action of this instrument. Even now there are points which remain obscure, for example the easy andibility of sounds when the iron disk is replaced by one of copper or aluminium. It is to be presumed that the movements of the disk then depend upon electric currents induced in it. If so, they would follow different laws from those governing the behaviour of a simply magnetised disk ; and in the case of iron complications would ensue from the cooperation of both causes.

Again, though this is partly a matter of definition, I am of opinion that the sensitiveness of the ear is best investigated with the ear free. When a telephone, pressed closely up, is employed, the situation is materially altered. For example, the natural resonances of the ear-passage must be seriously disturbed.

The above objections do not apply to some of Wien's determinations, where the ear was placed at a distance from the telephone and the vibrations of the plate were directly measured; and his conclusions must necessarily carry great weight. But I could not forget that my own experiments in 1894 had been carefully made, and I was desirous, if possible, of checking the results by some new method different from those previously employed either by Wien or myself. The difficulties of the problem are considerable; but it occurred to me that, so far as the important question of the dependence of sensitiveness upon pitch is concerned, they might be turned by calling to our aid the general principle of dynamical similarity *. Thus if vibrations are communicated to the air from the prongs of a tuning-fork, we are unable to calculate the theoretical counexion between the invisible aerial vibrations and the visible amplitude of the prongs. But if there are two precisely similar forks of different dimensions, and each communicating vibrations, a comparison may be effected. In the first place, if the material is the same, the times of vibration of the forks (regarded as uninfluenced by the air) are as the linear dimensions. And further, what is more important for our purpose, the

* The application of this principle to acoustical problems is discussed in 'Theory of Sound,' 2nd ed. \$ 381 . 
condensations at corresponding points in the surrounding air will be the same, provided the amplitudes of vibrations at the prongs be themselves in the proportion of the linear dimensions. Corresponding points are, of course, such as are similarly situated with respect to the vibrating forks, the distances from corresponding points of the forks being in proportion to the linear dimensions of the latter. Since times and distances are altered in the same proportion, velocities are unchanged. In conformity with this, the velocity-potentials in the two systems are as the linear dimensions.

It appears then that by means of the principle of similarity we can obtain aerial condensations which may be recognized to be equal in spite of a change of pitch. As has been said, equality occurs when the amplitudes of the solid vibrators are as the linear dimensions. In virtue of the principle of superposition as applicable to the small vibrations of either system, we are not limited to the case of equal condensations. The ratio of condensations can be inferred from the ratio of amplitudes by introduction of the factor expressing the linear magnification.

My first intention had been to use forks for the actual experiments. But apart from the difficulty of obtaining them of the necessary geometrical similarity over a sufficient range, it appeared that the communication of vibration to the air was inadequate. At a suitable distance there was danger that the sounds might prove inaudible. In connexion with this the difficulty of supporting the forks has to be considered. It is essential that no sound capable of influencing the results shall reach the ear by way of the supports, to which the principle of similarity can hardly be extended; and the danger of disturbance from this source increases if the direct communication of vibration to the air is too much enfeebled. On the other hand the arrangements must, if possible, be such as will render adequate the optically observed amplitude up to the point at which the ear is beginning to fail. We have in fact to steer as best we may between difficulties on opposite sides.

The requirements of the case seem to be best met by using thin open metal cans vibrating after the manner of bells. They were constructed by Mr. Enock from tin-plate or ferrotype plate and were maintained in vibration electrically. The wall is a simple cylinder and there is a flat bottom of similar material. In the case of the largest can, giving 85 complete vibrations per second, the height was 8 inches and 
the diameter 4.5 inches. During the vibration the circular bottom bends, and thus the can must not be beld fast round the lower circumference. Support was usually given at the centre only, by means of a short length of tube attached with solder. It is possible, however, as will be explained more fully later, to give support at four points of the lower circumference, or rather along two diameters of the base perpendicular to one another. These diameters are at $45^{\circ}$ to the line of the electromagnet by which the vibrations are maintained. In order to avoid communicating vibration, the metal handle of the can was attached to a large bung, resting upon a leaden slab, supported in its turn from the floor by a tall retort-stand. Before or after observations the bung could be lifted with the fingers and security taken that all the sound heard came direct from the vibrating can.

The bar electromagnet, by which the iron substance of the can is attracted, lies just below a diameter of the upper rim and is supported from the centre of the base. Since the electromagnet acts as an obstacle to the aerial vibrations in the region where they are strongest, care must be taken that in passing from one can to another geometrical similarity extends to the external form of the magnet and its accessories. The current was supplied by an interrupter-fork and usually both cans under comparison were driven (alternately) from the same fork, so that no question could arise with respect to the accuracy of the musical intervals. In constructing the cans the thickness of the plate employed was taken proportional to the other linear dimensions, but this alone would not suffice to secure an accurate tuning. The final adjust ment for the greatest possible response to the intermittent electric current was effected with wax, required only in small quantities.

Trouble was sometimes experienced from the intrusion of undesired tones. A shunt across the mercury break of the interrupter-fork, as employed by Helmholtz, was useful, and an improvement would often follow a readjustment of the position of the electromagnet. No observations were taken until false sounds had been rendered entirely subordinate, if not inaudible. In every case the ear was placed in the plane of the rim opposite the loops L (fig. 1), where the radial motion is greatest, two of which face the poles of the electromagnet. All four positions were ntilized, and either the iniensity of vibration or the common distance was varied until the average intensity was judged suitable. The intensity aimed at was such that the sound was just 
easily audible, but sometimes a little more was allowed. The amplitude of vibration at a loop $L$ was then measured by

Fig. 1.

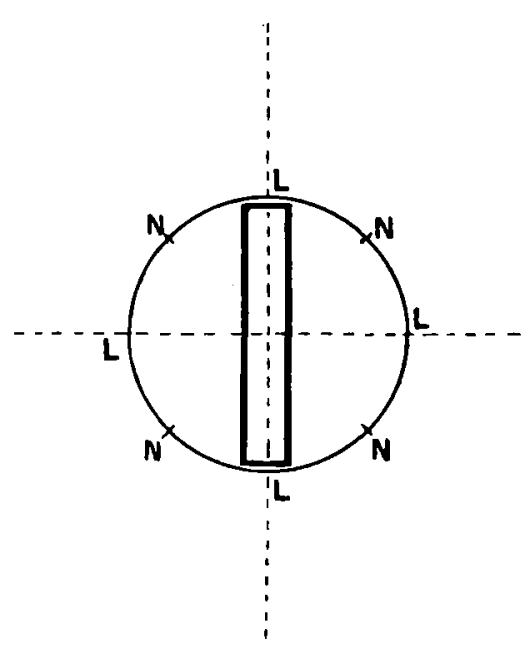

means of a microscope provided with a micrometer-scale and focussed upon starch grains carried by the rim. In passing to the can under comparison the distance of observation is no longer variable at pleasure but must be taken in proportion to the linear dimension. Thus if the second can be on half the scale of the first and sound the octave above, the distance must be halved. If, when listened to in the four positions, the sound is judged too strong or too faint, the vibration must be modified (by varying interposed resistance) until the former audibility is reproduced. The amplitude of vibration at a loop is then measured with a second microscope similar to the first. The distances of the ear, measured from the rim, varied from 8 inches to 24 inches. The sounds under comparison were usually estimated independently by Mr. Enook and myself. A slight tendency on my part to estimate the graver sounds as the louder was suspected, but the difference was of no importance. Other observers also have taken part occasionally, and there was sufficient repetition on different days to eliminate chance errors. It will suffice to record the mean results.

In the comparison of cans of dimensions in the ratio of $2: 1$ and inaking 128 and 256 vibrations per second, it was found 
that for equal audibility at distances in the ratio of $2: 1$ the radial amplitude of the larger can required to be $4 \cdot 0$ times that of the smaller. Equal aerial condensations at the points of observation require amplitudes in the ratio of $2: 1$, from which we infer that for equal audibilities the condensation needed at pitch 128 is the double of that needed at pitch 256 . In like manner observations with another pair of cans showed that the condensation needed at 256 was $1 \cdot 6$ of that needed at 512 vibrations per second. It did not appear feasible by this method to go to higher pitch, but the range could be extended at the other end. For this purpose the largest can already spoken of was constructed, whose dimensions relatively to the 128 can were as $3: 2$. In this case the interval was a Fifth, and the comparison showed that the condensation necessary for audibility at 85 per second was almost precisely the double of that needed at pitch 128 . So far no interval had been attempted exceeding the octave; but subsequently confirmation was obtained by a direct comparison between the cans vibrating 256 and 85 per second. With large intervals the difficulties are increased, as the amplitude of the smaller can is too minute for satisfactory measurement under the microscope.

Since the numbers have merely a relative value, we may call the condensation necessary for audibility at pitch 512 unity. The results are then summarized in the accompanying statement. It was rather to my surprise that I found my

$$
\begin{array}{c|r|r|r|r|}
\mathrm{N} \ldots \ldots & 512 & 256 & 128 & 85 \\
s \ldots \ldots & 1.0 & 1.6 & 3.2 & 6 \cdot 4
\end{array}
$$

former conclusion as to the small variation of sensitiveness in the octave $256-512$ substantially confirmed. Below 256 and especially below 128, it is evident that the sensitiveness of the ear falls off more rapidly; but even here the differences appear much less than those calculated by Wien from his own observations. I am much at a loss to explain the discrepancy. Although doubtless criticisms may be made, I should have supposed that both methods were good enough to yield fairly approximate results.

To give a general idea of the trend, a plot of the values of $s$ is given in fig. 2, the logarithm of the periodic time being taken as abscissa. It would appear that the minimum $s$, corresponding to maximum sensitiveness of the ear, would not be reached under 1024 vibrations per second, and perhaps 
not until an octave higher, in accordance with Wien's conclusions.

Fig. 2.

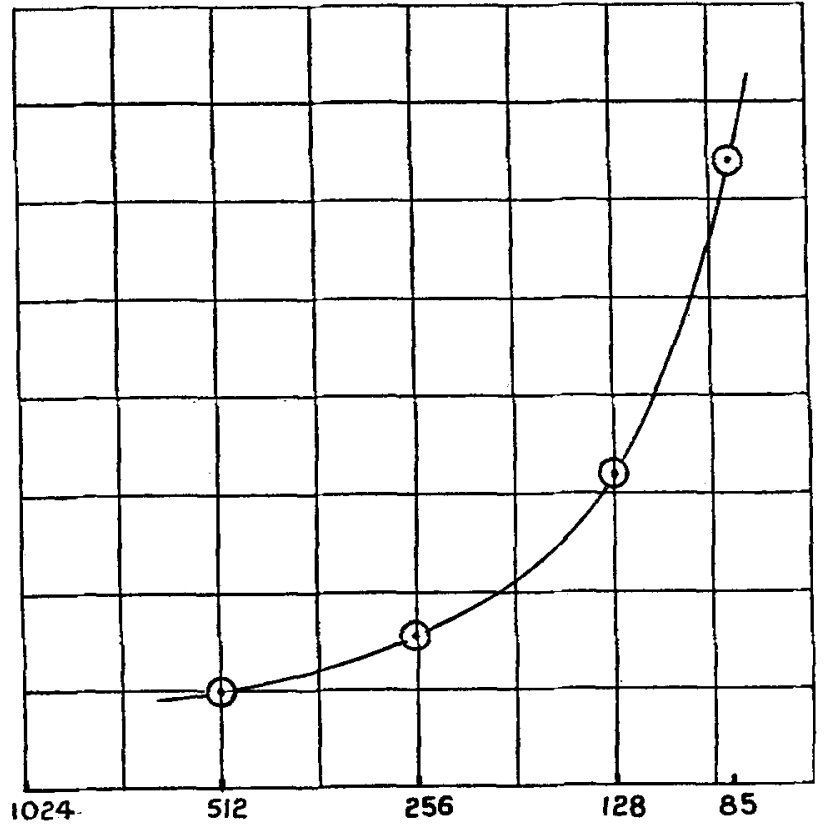

I take this opportunity of recording a few observations on the mode of vibration of these cans, although the resulta have no immediate connexion with the main subject of this note. The theory of the vibration of thin cylindrical shells vibrating without extension of the middle surface ${ }^{*}$ indicates two distinct types, of which one is excluded by the action of the plane disk forming the bottom of a can. The remaining type is defined by the equations

$$
\delta r=s z \sin s \phi, \quad a \delta \phi=z \cos s \phi, \quad \delta z=-s^{-1} a \sin s \phi, .
$$

in which $z$ is measured upwards from the bottom, and the angle $\phi$ is measured round the circumference, the radius being $\alpha . \delta r, a \delta \phi$, and $\delta z$ are the radial, circumferential, and axial displacements of the point whose equilibrium position is defined by $a, \phi, z$. When $z=0, i, e$. at the junction of the cylindrical and plane parts, $\delta r$ and $a \delta \phi$ vanish, but $\delta z$

* Proceedings Royal Society, xlv. p. 105, 1888 ; Scientific Papers, iii. p. 217. 
remains finite except when $\sin s \phi=0$. Thus if $\delta z$ be constrained to remain zero all round the circumference of the bottom, no vibration of this kind is possible; but the bottom may be supported at the places defined by $\sin s \phi=0$, which are situated under the nodes. $N$ (fig. 1) of the radial motion at the upper rim.

If, as in the above experiments, we limit ourselves to the principal vibration for which $s=2$, and if the height be $l$, we have for the maximum amplitudes

$$
\delta r_{z=l}=2 l, \quad a \delta \phi_{z=l}=l, \quad \delta z=\frac{1}{2} a . . .
$$

These relations were verified by observation under the microscope. The ratio of the maximum radial to the maximum circumferential motion at the upper rim was found to be almost exactly $2: 1$. The accurate observation of $\delta z$, as found at the bottom, was more difficult on account of its relative smallness. In the actual case $(85$ per second) $l=8$ ins., $2 a=4 \frac{1}{2}$ ins., so that $8 l / 2 a=14$. This is the theoretical ratio of the maximum radial motion at the rim to the maximum axial motion. By observation the mean number was 15 , as close to theory as could be expected.

Aug. 15, 1907.

LX. Secondary Röntgen Radiation in Air. By R. T. Beatty, M.A., B.E. (R.U.I.), Queen's College, Belfast, and University of Liverpool*.

$\mathrm{F}^{\text {ROM experiments made by Barklat, it seems that the }}$ constituents of a Röntgen beam are scattered approximately in equal proportions by certain elementary substances, while others transform the incident radiation into a more homogeneous beam which is more easily absorbed by matter than the primary radiation. Further, the nature of the radiation from all the elements examined is found to vary in a periodic manner with the atomic weight of the radiator.

In observing the behaviour of those solids which scatter radiations, the results are complicated by the fact that the different constituents of the secondary beam come from different thicknesses of the solid. Thus the radiations coming from deeper layers of the solid are robbed of their more absorbable constituents in emerging, and so perfect scattering of the primary beam is not to be expected.

Now in air the absorption of Röntgen radiation is very small, and as air is included in the category of scatterers

* Communicated by Professor. Wilberforce.

† Barkla, Phil. Mag. June 1906. 